\title{
Theoretical Study on Electronic Structure and Charge Transport Characteristics of Anthracene-based Host Material for Blue Organic Light-emitting Diode Application
}

\author{
Hyunbok Lee* \\ Department of Physics, Kangwon National University, Gangwon-do 24341, Republic of Korea
}

Received November 19, 2018; accepted November 21, 2018

\begin{abstract}
Organic light-emitting diodes (OLEDs) have received much attention for application the in next-generation display due to their many advantages. To increase the device performance of OLEDs, a host-dopant system in the emission layer has been used. In blue OLEDs, anthracene-based materials have been used as a host material. To understand the device behavior of OLEDs, a fundamental study on the electronic properties of organic semiconductors is necessary. In this study, theoretical calculations using density functional theory were performed to investigate the electronic structure and charge transport ability of 9,10-diphenyl-2-((3-trifluoromethyl)phenyl)anthracene (ATFP-Ph), 9,10-di([1,1'-biphenyl]-4(trifluoromethyl)phenyl)anthracene (ATFP-BiPh), and 9,10-di(naphthalene-2-yl)-2-(3-(trifluoromethyl)phenyl)anthracene (ATFP-Naph). All molecules have similar lowest unoccupied molecular orbital and highest occupied molecular orbital energy levels, and thus their charge injection abilities from the adjacent layer to the host material are similar. However, ATFP-Ph has significantly lower hole and electron reorganization energy $(\lambda)$ of 250 and $268 \mathrm{meV}$, compared to that of ATFP-BiPh which has hole and electron $\lambda$ of 334 and $320 \mathrm{meV}$, and ATFP-Naph which has hole and electron $\lambda$ of 324 and $361 \mathrm{meV}$, respectively. The origin of lower hole and electron $\lambda$ is analyzed via charge distribution on the molecule. The change in charge on the phenyl moiety in ATFP-Ph during hole and electron injection is much smaller than the biphenyl moiety in ATFP-BiPh and the naphthyl moiety in ATFP-Naph, indicating low electron-phonon coupling. This low hole and electron $\lambda$ of ATFP-Ph yield high hole and electron hopping rates, which result in the higher device performance of OLEDs.
\end{abstract}

Keywords: Organic light-emitting diode, Reorganization energy, Density functional theory, Host material

\section{Introduction}

Organic light-emitting diodes (OLEDs) have received great attention due to their wide angle viewing, thin thickness, high contrast, and mechanical flexibility [1]. Since the first report of a multilayer structure of OLEDs by Tang [2], device performance of OLEDs has been significantly improved, and now they utilized in the display for televisions and smartphones. To increase the quantum efficiency, a host-dopant system is used for light emission. The holes and electrons are transported to the host material, then generated excitons transfer to the dopant material to emit light with the desired color.

Well-aligned energy levels with adjacent layers and high and ambipolar charge mobility are required for an efficient host material [3]. Anthracene-based materials are widely used [e.g. 9,10-bis(2-naphthyl)anthracene (ADN)] as a host

*Corresponding author

E-mail: hyunbok@kangwon.ac.kr material [4]. Recently, 9,10-diphenyl-2-((3-trifluoromethyl) phenyl)anthracene (ATFP-Ph), 9,10-di([1,1'-biphenyl]-4(trifluoromethyl)phenyl)anthracene (ATFP-BiPh), and 9,10di(naphthalene-2-yl)-2-(3(trifluoromethyl)phenyl) anthracene (ATFP-Naph) were synthesized, and OLEDs were characterized using these host materials and a 4,4'-bis[4(di-p-tolylamino)styryl] biphenyl (DPAVBi) dopant material [5]. Among these, OLEDs with ATFP-Ph showed much higher device performance than OLEDs with ATFP$\mathrm{BiPh}$ and ATFP-Naph. This could be attributed to the superior charge transport characteristics of ATFP-Ph over ATFP-BiPh and ATFP-Naph. However, the origin of different device performance is still not well understood.

In this study, we investigated the electronic structure and charge transport ability of ATFP-Ph, ATFP-BiPh, and ATFP-Naph host materials using density functional theory (DFT) calculations. Frontier energy levels, molecular orbital, reorganization energy $(\lambda)$, and charge distribution were calculated. The origin of higher device performance of OLEDs with ATFP-Ph is discussed with a comparison 
of charge hopping rates of host materials based on Marcus theory.

\section{Experimental details}

DFT calculations were performed at a single molecule of ATFP-Ph, ATFP-BiPh, and ATFP-Naph. Because organic semiconductors interact with each other with weak van der Waals type forces, the intermolecular coupling is small and thus the electronic structure of a single molecule well approximates that of a film [6]. In this study, a hybrid functional of Becke three parameters exchange and LeeYang-Parr correlation (B3LYP) and a 6-31G(d,p) basis set implemented in a GAUSSIAN 09 package were used to calculate the total energy, geometry optimization, and vibrational frequency calculations [7-10].

Hopping is the major mechanism of charge transport in organic semiconductors during device operation. In this case, the semi-classical Marcus theory can be applied to explain the charge transport process [11]. The charge hopping rate $k$ can be written as the following equation:

$$
\mathrm{k}=\frac{4 \pi^{2}}{h} V^{2} \frac{1}{\sqrt{4 \pi \lambda k_{B} T}} \exp \left(-\frac{\lambda}{4 k_{B} T}\right)
$$

where $V$ is the transfer integral, $h$ is the Planck constant, $T$ is the temperature in Kelvin, and $k_{B}$ is the Boltzmann constant. Since $h, T$, and $k_{\mathrm{B}}$ are constant in any given condition, only $\lambda$ and $V$ are critical parameters in $k$ (i.e. mobility). To increase $k$, a decrease in $\lambda$ and an increase in $V$ are necessary. However, in amorphous or polycrystalline organic semiconductors, the intermolecular geometry of molecules would not be tightly packed and the mobility would be dominated by $\lambda$ [12]. Thus, only $\lambda$ was considered in this study.

The $\lambda$ of molecules are composed of internal $\lambda$ and external $\lambda$. However, it is known that organic semiconductors' external $\lambda$ does not significantly influence the total $\lambda$, therefore we proceeded on the assumption that internal $\lambda$ molecules are the only significant contributors to $k$ [13]. Thus, $\lambda$ can be calculated using four-point methods equations, as follows $[14,15]$ :

$$
\lambda=\lambda_{1}+\lambda_{2}=\left[E^{*}\left(Q_{0}\right)-E^{*}\left(Q^{*}\right)\right]+\left[E_{0}\left(Q^{*}\right)-E_{0}\left(Q_{0}\right)\right]
$$

where $E^{*}\left(Q_{0}\right)$ is the charged state energy in the neutral state geometry, $E^{*}\left(Q^{*}\right)$ is the charged state energy in the charged state geometry, $E_{0}\left(Q^{*}\right)$ is the neutral state in the charged state geometry, and $E_{0}\left(Q_{0}\right)$ is the neutral state energy in the neutral state geometry.

\section{Results and discussion}

Figure 1 shows the optimized geometry of ATFP-Ph,

(a)

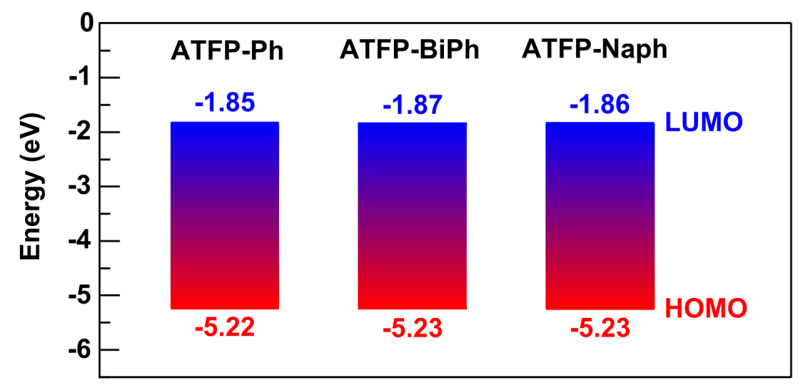

(b)

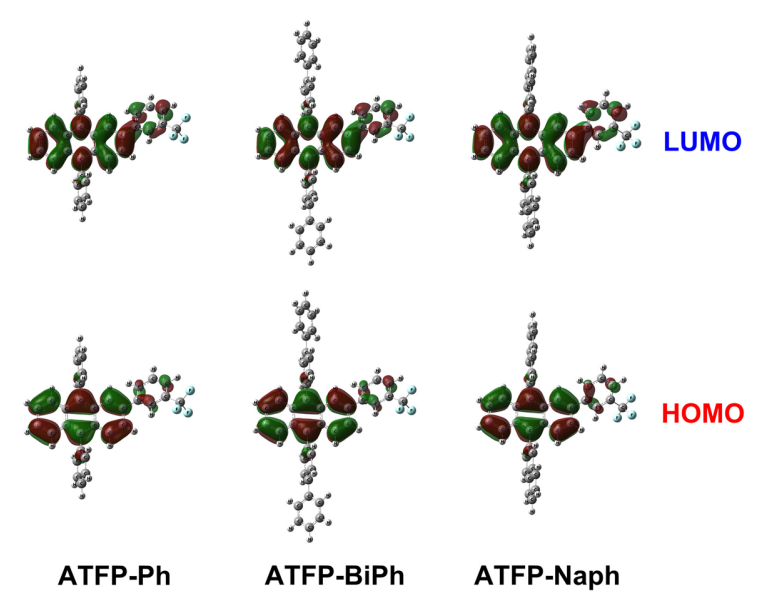

Figure 2. (a) Calculated LUMO and HOMO energy levels and (b) LUMO and HOMO distribution of ATFP-Ph, ATFP-BiPh, and ATFP -Naph.
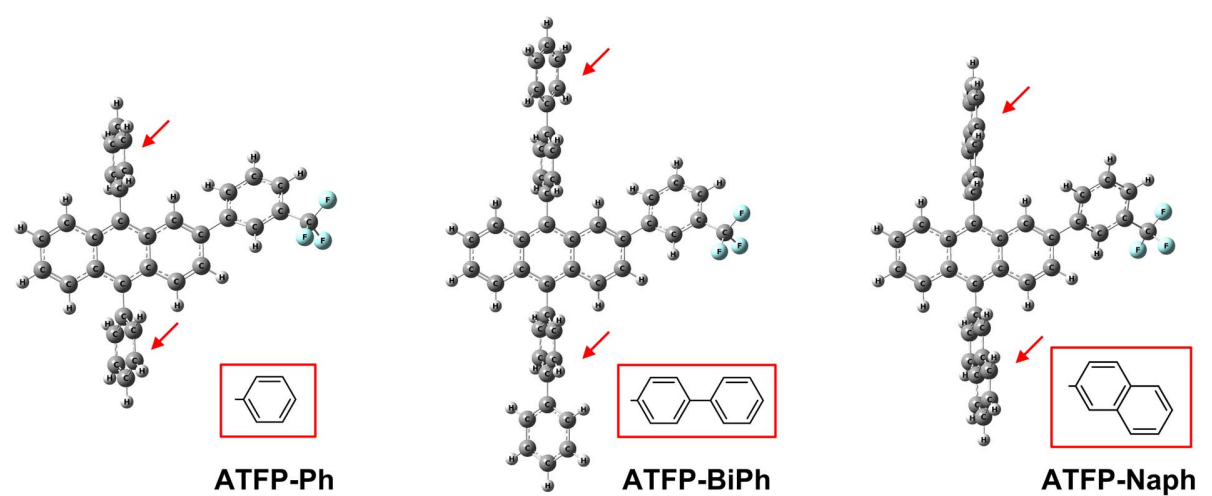

Figure 1. Optimized geometry of ATFP-Ph, ATFP-BiPh, and ATFP-Naph with DFT calculations. 
ATFP-BiPh, and ATFP-Naph derived from DFT calculations. Anthracene and trifluoromethyl-phenyl moieties are common in the three molecules, but the side groups vary as phenyl, biphenyl, and naphthyl moieties. The electronwithdrawing property of trifluoromethyl-methyl moiety lowers the energy levels to match the charge transport levels of the adjacent layers in OLEDs. The side groups are indicated with the red arrow and the chemical formula is shown in the inset. These side groups are flexibly connected with the anthracene moiety, and thus the geometry of side groups can be easily changed during charge transfer.

Figure 2(a) shows the lowest unoccupied molecular orbital (LUMO) and highest occupied molecular orbital (HOMO) energy levels of ATFP-Ph, ATFP-BiPh, and ATFP-Naph molecules determined by DFT calculations. The calculated LUMO and HOMO levels of the three molecules are almost the same. The LUMO and HOMO levels of ATFP-Ph are -1.85 and $-5.22 \mathrm{eV}$, the values of ATFP-BiPh are -1.87 and $-5.23 \mathrm{eV}$, and the values of ATFP-Naph are -1.86 and $-5.23 \mathrm{eV}$, respectively. This result is in good agreement with the values measured by
Table I. Calculated $\lambda$ of ATFP-Ph, ATFP-BiPh, and ATFPNaph.

\begin{tabular}{ccccccc}
\hline \hline & \multicolumn{2}{c}{ ATFP-Ph } & \multicolumn{2}{c}{ ATFP-BiPh } & \multicolumn{2}{c}{ ATFP-Naph } \\
\cline { 2 - 7 } & Hole & Electron & Hole & Electron & Hole & Electron \\
\hline$\lambda_{1}(\mathrm{meV})$ & 133 & 138 & 197 & 171 & 186 & 201 \\
$\lambda_{2}(\mathrm{meV})$ & 116 & 130 & 137 & 149 & 138 & 160 \\
$\lambda(\mathrm{meV})$ & 250 & 268 & 334 & 320 & 324 & 361 \\
\hline
\end{tabular}

cyclic voltammetry and UV-vis absorption reported in the literature [5]. Figure 2(b) shows the LUMO and HOMO distribution of ATFP-Ph, ATFP-BiPh, and ATFP-Naph. Both LUMO and HOMO mainly originate from the anthracene and trifluoromethyl-phenyl moieties. The side phenyl, biphenyl, and naphthyl moieties contribute little to the LUMO and HOMO. Considering that, all molecules have similar LUMO and HOMO levels. In OLEDs, electrons are transported from the electron transport layer (ETL) and holes are transported from the hole transport layer (HTL) to the host layer. For efficient charge transport, the energetic offset between the HOMO levels of HTL/host and the LUMO levels of ETL/host should be minimized. In
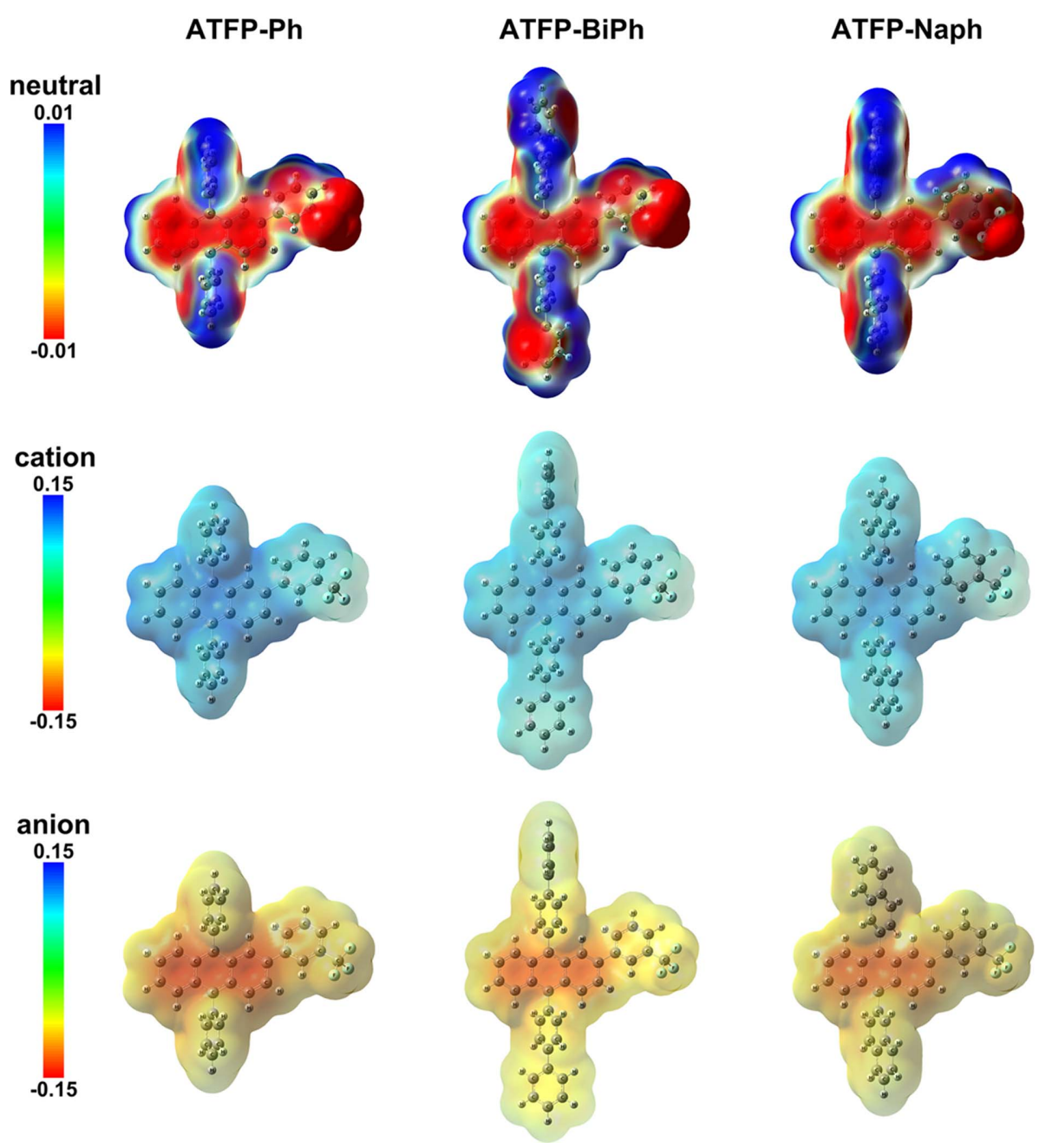

Figure 3. Charge distribution based on electrostatic potential of neutral, cation, and anion state of ATFP-Ph, ATFP-BiPh, and ATFP-Naph (scale bar: $e$ in atonic unit). 
these host materials, the LUMO and HOMO levels are almost the same, and the charge injection abilities are similar. Therefore, the difference in device performance cannot be attributed to energy level differences.

To examine the $k$ of host materials, their hole and electron $\lambda$ were calculated. Table I shows the calculated $\lambda$ of ATFP-Ph, ATFP-BiPh, and ATFP-Naph. For ATFP-Ph, hole $\lambda$ and electron $\lambda$ was determined to be 250 and $268 \mathrm{meV}$, respectively. The balanced hole and electron $\lambda$ are related to the ambipolar charge transport property. On the other hand, for ATFP-BiPh, hole $\lambda$ and electron $\lambda$ was determined to be 334 and $320 \mathrm{meV}$, respectively. Thus, both hole $\lambda$ and electron $\lambda$ of ATFP-BiPh are higher than those of ATFP-Ph. In addition, hole $\lambda$ and electron $\lambda$ of ATFP-Naph were determined to be 324 and $361 \mathrm{meV}$, respectively. These values are higher than ATFP-Ph, but similar to ATFP-BiPh. Since $\lambda$ exists in the exponential term in $k$ according to Marcus theory, even a small change in $\lambda$ significantly affects $k$. If we assume that the $V$ is similar in all molecules, the lower hole $\lambda$ and electron $\lambda$ of ATFP-Ph cause ATFP-Ph to exhibit 2.6 times higher hole $k$ and 1.8 times higher electron $k$ than ATFP-BiPh and 2.3 times higher hole $k$ and 2.9 times higher electron $k$ than ATFP-Naph, respectively. Thus, the low hole $\lambda$ and electron $\lambda$ of ATFP-Ph leads to superior mobility over other two materials. This is the cause of higher device performance in OLEDs [5].

To understand the origin of different $\lambda$ of host materials, charge distribution based on electrostatic potential was calculated. Figure 3 shows the charge distribution of the neutral, cation, and anion states of ATFP-Ph, ATFP-BiPh, and ATFP-Naph. The blue and red color indicate positive and negative charge polarity, respectively. In the charged states (cation and anion), it is observed that the hole and electron distribution on anthracene is higher in ATFP-Ph than in the other two materials. In other words, the change in charge distribution in the side group during the charge injection of both hole and electron is smaller in ATFP-Ph than the other two materials. This directly relates to the strength of electron-phonon coupling.

To analyze the charge distribution in detail, Mulliken charge analysis was performed. Since the anthracene and trifluoromethyl-phenyl moieties are common in the molecules, only charge distributions in phenyl, biphenyl, and naphtyl moieties were compared. Table II shows the

Table II. Mulliken charge analysis of a phenyl moiety in ATFP-Ph, biphenyl moiety in ATFP-BiPh, and naphthyl moiety in ATFP-Naph.

\begin{tabular}{cccc}
\hline \hline & ATFP-Ph & ATFP-BiPh & ATFP-Naph \\
\hline Neutral $(e)$ & -0.0165 & -0.0170 & -0.0188 \\
Cation $(e)$ & 0.1189 & 0.1704 & 0.1567 \\
Anion $(e)$ & -0.1201 & -0.1749 & -0.1922 \\
Cation-Neutral $(e)$ & 0.1354 & 0.1873 & 0.1755 \\
Anion-Neutral $(e)$ & -0.1036 & -0.1579 & -0.1735 \\
\hline
\end{tabular}

calculated Mulliken charge of a phenyl moiety in ATFP$\mathrm{Ph}$, biphenyl moiety in ATFP-BiPh, and naphthyl moiety in ATFP-Naph. In the neutral, cation, and anion states, the phenyl moiety in ATFP-Ph contains $-0.0165 e, 0.1189 e$, and $-0.1201 e$, respectively ( $e$ : elementary charge). On the other hand, in the neutral, cation, and anion states, the biphenyl moiety in ATFP-BiPh contains -0.0170e, 0.1704e, and $0.1749 e$, and the naphthyl moiety in ATFP-Naph contains $-0.0188 e, 0.1567 e$, and $-0.1922 e$, respectively. Thus, the difference in charge on the phenyl moiety in ATFP between the cation and neutral states is $0.1354 e$, and between the anion and neutral states is $-0.1036 e$. Meanwhile, the difference of the charge on the biphenyl moiety in ATFP-BiPh between the cation and neutral states is $0.1873 e$, and between the anion and neutral states is 0.1579e. In ATFP-Naph, the difference in charge on the naphthyl moiety between the cation and neutral states is $0.1755 e$, and between the anion and neutral states is $0.1735 e$. Thus, in both hole and electron injection to the host material, the change in charge in the side group is much smaller in ATFP-Ph than in ATFP-BiPh and ATFPNaph. This trend is in accord with the magnitude of hole and electron $\lambda$ and implies that the interaction between electron and phonon in the side group is stronger in ATFP$\mathrm{Ph}$ than in the other two materials. The strong interaction between electron and phonon results in strong geometrical change during hole and electron transfer, and thus $\lambda$ is smaller in ATFP-Ph than in the other two materials. It also results in higher performance of OLEDs with ATFP-Ph, compared to OLEDs with ATFP-BiPh or ATFP-Naph.

\section{Conclusions}

In this study, the electronic structure and charge transport properties of anthracene-based host materials, ATFP-Ph, ATFP-BiPh, and ATFP-Naph, were investigated using DFT calculations. All molecules have similar LUMO and HOMO levels, and thus charge injection to these host materials would be similar in OLEDs. However, both hole and electron $\lambda$ of ATFP-Ph are significantly smaller (250 and $268 \mathrm{meV}$ ) than those values of ATFP-BiPh (334 and $320 \mathrm{meV}$ ) and ATFP-Naph (324 and $361 \mathrm{meV}$ ), respectively. The origin of the smaller $\lambda$ was analyzed with charge distribution on the side groups. During hole and electron injection to the host material, the phenyl moiety in ATFP$\mathrm{Ph}$ exhibited a smaller change in charge than the other two materials. Thus, electron-phonon coupling would be smaller in ATFP-Ph than in the other two host materials and was determined to be the origin of the superior device performance of OLEDs with ATFP-Ph.

\section{Acknowledgements}

This study was supported by National Research Foundation of Korea (NRF-2018R1D1A1B07051050). 


\section{References}

[1] B. Geffroy, P. le Roy, and C. Prat, Polym. Int. 55, 572 (2006).

[2] C. W. Tang and S. A. VanSlyke, Appl. Phys. Lett. 51, 913 (1987).

[3] Y. Tao, C. Yang, and J. Qin, Chem. Soc. Rev. 40, 2943 (2011).

[4] M. Zhu and C. Yang, Chem. Soc. Rev. 42, 4963 (2013).

[5] S. W. Kwak, K. M. Lee, J. E. Lee, J. Yoo, Y. Yi, H. Kwon, H. Lee, M. H. Park, and Y. Chung, Appl. Sci. Basel 7, 1109 (2017).

[6] D. R. T. Zahn, G. N. Gavrila, and G. Salvan, Chem. Rev. 107, 1161 (2007)

[7] M. J. Frisch, G. W. Trucks, H. B. Schlegel, G. E. Scuseria, M. A. Robb, J. R. Cheeseman, G. Scalmani, V. Barone, B. Mennucci, G.A. Petersson, H. Nakatsuji, M. Caricato, X. Li, H. P. Hratchian, A. F. Izmaylov, J. Bloino, G. Zheng, J. L. Sonnenberg, M. Hada, M. Ehara, K. Toyota, R. Fukuda, J. Hasegawa, M. Ishida, T. Nakajima, Y. Honda, O. Kitao, H. Nakai, T. Vreven, J. A. J. Montgomery, J. E. Peralta, F. Ogliaro, M. Bearpark, J. J. Heyd, E. Brothers, K. N. Kudin, V. N. Staroverov, R. Kobayashi, J. Normand, K. Raghavachari, A. Rendell, J. C. Burant, S. S. Iyengar,
J. Tomasi, M. Cossi, N. Rega, J. M. Millam, M. Klene, J. E. Knox, J. B. Cross, V. Bakken, C. Adamo, J. Jaramillo, R. Gomperts, R. E. Stratmann, O. Yazyev, A. J. Austin, R. Cammi, C. Pomelli, J. W. Ochterski, R. L. Martin, K. Morokuma, V. G. Zakrzewski, G. A. Voth, P. Salvador, J. J. Dannenberg, S. Dapprich, A. D. Daniels, Ö. Farkas, J. B. Foresman, J. V. Ortiz, J. Cioslowski, and D. J. Fox, Gaussian 09, Gaussian Inc. Wallingford CT, 2009.

[8] A. D. Becke, J. Chem. Phys. 98, 5648 (1993).

[9] C. Lee, W. Yang, and R.G. Parr, Phys. Rev. B 37, 785 (1988).

[10] S. F. Sousa, P. A. Fernandes, and M. J. Ramos, J. Phys. Chem. A 111, 10439 (2007).

[11] R. A. Marcus, Rev. Mod. Phys., 65, 599 (1993).

[12] P. Cias, C. Slugovc, and G. Gescheidt, J. Phys. Chem. A 115, 14519 (2011).

[13] D. P. McMahon and A. Troisi, J. Phys. Chem. Lett. 1, 941 (2010).

[14] L. Wang, G. Nan, X. Yang, Q. Peng, Q. Li, and Z. Shuai, Chem. Soc. Rev. 39, 423 (2010).

[15] J. L. Brédas, D. Beljonne, V. Coropceanu, and J. Cornil, Chem. Rev. 104, 4971 (2004). 